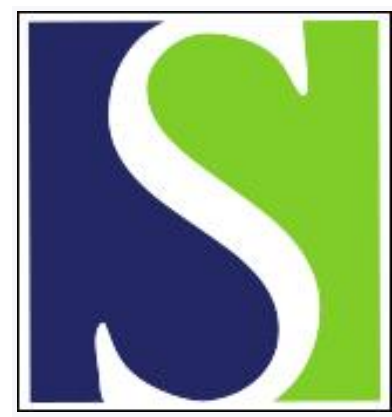

Scand J Work Environ Health 2014;40(6):582-596

https://doi.org/10.5271/sjweh.3449

Published online: 13 Aug 2014, Issue date: 01 Nov 2014

Efficacy of an internet-based problem-solving training for teachers: results of a randomized controlled trial

by Ebert DD, Lehr D, Boß L, Riper H, Cuijpers P, Andersson G, Thiart H, Heber E, Berking M

Internet-based interventions may be a promising approach to address the adverse effects of occupational stress. However, evidence from RCT for internet-based occupational mental-health interventions is scarce. This study may be the first that evaluates a internet-based guided self-help intervention aimed at reducing depressive symptoms among teachers (as well as employees in general)

Affiliation: Leuphana University Lüneburg, Innovation Incubator, Division Health Trainings Online, Rotenbleicher Weg 67, 21335 Lüneburg, Germany. ebert@inkubator.leuphana.de

Refers to the following texts of the Journal: 2006;32(6):443-462 2009;35(1):7-18

The following articles refer to this text: $2015 ; 41(2): 164-174$; 2016;42(5):382-394; 2018;44(2):171-182; 2019;45(6):560-576

Key terms: depression; depressive symptom; internet-based; mental health; occupational health; randomized controlled trial; RCT; teacher; training; web-based

This article in PubMed: www.ncbi.nlm.nih.gov/pubmed/25121986

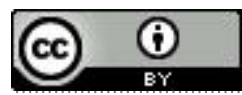




\title{
Efficacy of an internet-based problem-solving training for teachers: results of a randomized controlled trial
}

\author{
By David Daniel Ebert, PhD, 1, 2, 3, 4 Dirk Lehr, PhD, ${ }^{1,2}$ Leif Boß, MSc, ${ }^{2}$ Heleen Riper, PhD, ,2, 5, Pim Cuijpers, \\ PhD, ${ }^{6}$ Gerhard Andersson, PhD, ${ }^{7,8}$ Hanne Thiart, MSc, ${ }^{2}$ Elena Heber, MSc, ${ }^{2}$ Matthias Berking, PhD ${ }^{2,4}$
}

\begin{abstract}
Ebert DD, Lehr D, Boß L, Riper H, Cuijpers P, Andersson G, Thiart H, Heber E, Berking M. Efficacy of an Internetbased problem-solving training for teachers: Results of a randomized controlled trial. Scand J Work Environ Health. 2014;40(6):582-596. doi:10.5271/sjweh.3449
\end{abstract}

\begin{abstract}
Objective The primary purpose of this randomized controlled trial (RCT) was to evaluate the efficacy of internet-based problem-solving training (iPST) for employees in the educational sector (teachers) with depressive symptoms. The results of training were compared to those of a waitlist control group (WLC).

Methods One-hundred and fifty teachers with elevated depressive symptoms (Center for Epidemiologic Studies Depression Scale, CES-D $\geq 16$ ) were assigned to either the iPST or WLC group. The iPST consisted of five lessons, including problem-solving and rumination techniques. Symptoms were assessed before the intervention began and in follow-up assessments after seven weeks, three months, and six months. The primary outcome was depressive symptom severity (CES-D). Secondary outcomes included general and work-specific self-efficacy, perceived stress, pathological worries, burnout symptoms, general physical and mental health, and absenteeism.

Results iPST participants displayed a significantly greater reduction in depressive symptoms after the intervention $(\mathrm{d}=0.59,95 \%$ CI $0.26-0.92)$, after three months $(\mathrm{d}=0.37,95 \%$ CI $0.05-0.70)$ and after six months $(\mathrm{d}=0.38$, 95\% CI 0.05-0.70) compared to the control group. The iPST participants also displayed significantly higher improvements in secondary outcomes. However, workplace absenteeism was not significantly affected.
\end{abstract}

Conclusion iPST is effective in reducing symptoms of depression among teachers. Disseminated on a large scale, iPST could contribute to reducing the burden of stress-related mental health problems among teachers. Future studies should evaluate iPST approaches for use in other working populations.

Key terms depression; depressive symptom; mental health; occupational health; RCT; web-based

Occupational stress has been implicated as a risk factor for mental health problems, such as depression (1), impaired sleep (2) and early retirement (3). In particular, depression accounts for a considerable burden of disease for individuals, society, and employers $(4,5)$. Depressive symptoms are highly prevalent in working populations, with a 12 -month prevalence rate of $7.1 \%$ for men and $6.2 \%$ for women $(6,7)$. Depression is associated with considerable costs due to productivity loss and absenteeism $(8,9)$, and it negatively affects workplace safety $(10)$.
Recently, internet-based interventions for working populations were introduced as a promising approach to address the adverse effects of occupational stress $(11,12)$. Internet-based interventions are advantageous because they: (i) are easily accessible at any time and place, (ii) assure anonymity when participants want to avoid stigmatization or self-disclosure in group settings, (iii) allow participants to work at their own pace and review materials as often as they want, and (iv) may reach affected employees earlier than traditional mental

\footnotetext{
1 Joint first authorship.

2 Leuphana University, Innovation Incubator, Division Health Trainings Online, Lüneburg, Germany

3 Philipps University Marburg, Department of Psychology; Clinical Psychology and Psychotherapy, Marburg, Germany

4 Institute for Psychology, Division for Clinical Psychology and Psychotherapy, University of Erlangen-Nuremberg, Erlangen, Germany.

5 GGZ inGeest, Regional Mental Health Service Centre, VU University Medical Centre, Amsterdam, the Netherlands

6 Department of Clinical Psychology and EMGO Institute for Health and Care Research, VU University, Amsterdam, The Netherlands

7 Department of Behavioral Sciences and Learning, Linköping University, Sweden

8 Department of Clinical Neuroscience, Karolinska Institute, Stockholm, Sweden.
}

Correspondence to: David Daniel Ebert, Leuphana University Lüneburg, Innovation Incubator, Division Health Trainings Online, Rotenbleicher Weg 67, 21335 Lüneburg, Germany. [email: ebert@inkubator.leuphana.de] 
health services, hence preventing the onset of more severe mental health problems $(13,14)$.

Internet-based interventions have been shown to be effective in clinical applications, including the treatment of depression (15-17), anxiety (18), and sleep disorders (19). In particular, internet-based interventions for depression that include support from a healthcare professional (guided self-help) lead to greater effects and stronger adherence than self-help treatments on their own (15, 17). Internet-based interventions are also effective in changing negative health behaviors (eg, reducing alcohol consumption) (20), although mixed results were obtained for smoking cessation (21). Internet-based psychotherapy has been investigated in a large number of randomized controlled trials (RCT) (16-18). However, only a few interventions have been developed and evaluated to address the specific needs of working populations.

Evidence from RCT for internet-based occupational mental-health interventions is scarce. In what may be the first RCT of an internet-based stress management training, Zetterqvist and colleagues (11) found that an internet self-help intervention could be effective in reducing symptoms of stress, anxiety, and depression. In another study, computerized cognitive behavioral therapy for employees with recent stress-related absenteeism was found to be effective in reducing depression post-treatment compared to conventional care, but it was not found to be effective at the three-month follow-up (22). Ruwaard and colleagues (12) demonstrated that an e-mail-based cognitive behavioral treatment for work-related stress was more effective in reducing stress, depression, and anxiety than a waiting control condition. However, e-mail-based interventions might require a high degree of (health) literacy, and they do not make full use of the advantages of website-driven interventions, such as interactivity and multimedia interfaces, which can improve adherence, efficiency and cost-effectiveness. Another study evaluating a self-help online resilience training for sales managers observed no effects in terms of depression or productivity (23). Lastly, the efficacy of a mindfulness-based intervention at the workplace was investigated by comparing an online virtual to an in-person classroom. Interestingly, that study found no differences regarding the reduction of depression between the two environments. However, the online group had a significantly lower attrition rate compared to the face-to-face intervention (24). To the best of our knowledge, there has been no published study to evaluate internet-based guided self-help interventions in an occupational context with depressive symptoms as the primary outcome.

In contrast to internet-based interventions, traditional in-person stress management interventions have much stronger evidence, as demonstrated in several reviews and meta-analyses (25-27). When designing internet interventions for employees, some challenges and findings from this field should be considered. For example, Richardson and Rothstein (26) analyzed 19 RCT that considered the general working population, in which participants did not have a psychiatric diagnosis or stress-related somatic disorder. One of the main findings was that cognitive-behavioral interventions were more effective than other approaches (eg, relaxation, multimodal, organizational). Interventions with one or two treatment components were more effective than broader interventions with three or more components. Furthermore, shorter interventions were more effective than longer ones. Van der Klink and colleagues (27) observed the strongest effects for anxiety symptoms and the weakest for depressive symptoms. Similarly, Martin and colleagues (25) found that different types of interventions had a positive but small effect on depressive symptoms, which leaves room for improvement in terms of the design of effective treatments to reduce depression in a workplace setting.

Interventions based on problem-solving therapy (PST) have shown to be effective in reducing depression and several other mental health problems (28). According to D'Zurilla and Nezu (29), PST is based on the assumption that ineffective coping behavior causes psychopathology. Adverse health effects, especially depression and the creation of further problems, are expected if a person is not able to resolve stressful problems. PST aims to increase problem-solving skills and facilitate successful problem solving. As a consequence, a reduction in depression is expected. Besides this, worrying about problems should decrease and self-efficacy should increase as participants experience their ability to cope with problems and stressful situations more successfully.

The purpose of this study was to evaluate the efficacy of internet-based problem-solving training (iPST) for teachers with a heightened level of depressive symptoms. We chose this occupational group because school teachers face a high risk of work-related mental health issues and mood disorders $(3,30,31)$.

The primary objective was to test whether participating in iPST would lead to a greater reduction in depressive symptom severity compared to a waitlist control group (WLC). We further hypothesized that compared to the WLC group, participants in the iPST group would be more likely to report a reliable change in depressive symptom severity and be classified as being free of symptoms after having completed the iPST. Additionally, we explored whether the iPST group fared better than the WLC group in terms of secondary outcomes, such as perceived stress, worry, problem-solving skills, work-related and general self-efficacy, health-related quality of life, and work absenteeism. 


\section{Methods}

\section{Study design}

We evaluated the efficacy of iPST in a RCT including 150 participants with elevated symptoms of depression. Participants were randomly assigned to the intervention or WLC group. Self-reported outcome assessments were assessed at baseline (T1) and seven weeks (T2), three months (T3), and six months (T4) after randomization. The sample size was calculated to be able to detect a moderate effect at the post-treatment time point based on a power $(1-\beta)$ of 0.80 in a two-tailed test, $\alpha=0.05$. Participants who met the study criteria and provided informed consent were randomly allocated to either the iPST or WLC group. The groups were randomized by an independent researcher using a computer-based random integer generator (randlist). Participants of the iPST group received access to the training immediately after randomization, participants of the control group received access to iPST after the six-month follow-up. The Ethical Committee of the University of Marburg approved all procedures involved in the study which were consistent with generally accepted standards of ethical practice (reference number 2012-06K). The trial was registered at ISRCTN15635876.

\section{Participants and recruitment}

Participants were recruited from April 2012 to January 2013 with different recruitment strategies: (i) all over Germany, regional associations of school psychologists and educational institutes for teacher training were contacted and asked to relay information material about the study to potential participants; (ii) an invitation for study participation was posted on several online teacher forums; and (iii) announcements were made at several conferences concerning health promotion for teachers. To be eligible for the study, participants had to meet the following criteria: (i) a score of $\geq 16$ on the Center for Epidemiologic Studies Depression Scale (CES-D) $(32,33)$, (ii) be a working teacher, (iii) have sufficient German language (reading and writing) skills, (iv) have no notable suicidal risk as indicated by a score of $<2$ on item 9 of the Beck Depression Inventory (BDI) (34); (2="I'd like to kill myself", $3=$ ="I'd kill myself if I had a chance"). To maximize the external validity of the findings, there were no further exclusion criteria. The cut-off score for clinically relevant symptoms of depression in the German version of the CES-D is 23 whereas a cut-off score of 16 has been recommended for indicated prevention settings (32). As we aimed to reach employees suffering from elevated symptoms of depression and not restrict the intervention to employees suffering from mood disorders, we chose a cut-off score of 16. Individuals interested in participating in the study received notification via e-mail and were asked to complete an online screening questionnaire.

\section{Intervention}

Internet-based problem-solving training (iPST). The iPST is based on an empirically evaluated Dutch online-based intervention "Alles onder controle" (everything under control) $(35,36)$. The intervention was translated from Dutch into German and adapted for use by teachers. The iPST is composed of five lessons, in which participants acquire different problem-solving techniques. Additionally, the training includes components for behavioral activation with respect to important values in life. The following components were added to the original program: techniques for coping with rumination, video introductions for each lesson produced by a nationally known expert in mental health training for teachers and example teacher characters who depict the targeted problems and demonstrate implementation of problemsolving techniques in a variety of situations.

The iPST is structured as follows. First, participants describe what really matters to them (eg, values, lifegoals). Second, the participants write down their current worries and problems, which are then divided into three categories: unimportant, important but solvable, and unsolvable problems. Third, for each of the three types of problems, a different strategy is developed to either solve or cope with the problem if it is unimportant or unsolvable. The solvable problems are approached by a six-step procedure: describing the problem, brainstorming possible solutions, choosing the best solution, making a plan for carrying out the solution, actually carrying out the solution, and evaluating the success. Unsolvable and unimportant problems are handled by different coping techniques for rumination. Participants were advised to conduct one lesson per week and practice problem-solving techniques between lessons. Within 48 hours, participants received personalized written feedback from an eCoach on the exercises they had completed. The eCoaches were psychologists and trained master's-level psychology students who followed feedback guidelines according a standardized manual. Guidance was conceptualized according to a theoretical model for providing guidance in eHealth interventions (37). Thus, guidance was aimed at improving adherence to the web-based intervention, and the eCoaches were supervised to ensure that they did not teach techniques that go beyond those of the internet-based intervention.

Waitlist control (WLC). Teachers on the waiting list received no online intervention but had full access to treatment as offered by the workplace occupational health management programs and routine mental health 
services. Additionally, these teachers were granted access to iPST six months after the randomization.

\section{Primary outcome measure}

Depressive symptoms. The primary outcome was the level of depressive symptoms, as measured by the widely used CES-D $(32,33)$. This scale consists of 20 items. Subjects rate the frequency of symptoms during the past week on a 4-point Likert-scale $(0=$ rarely, less than one day, $1=$ sometimes, $2=$ more often, $3=$ most of the time, 5-7 days) (eg, "During the past week I felt sad"). The item values can be summarized to a total score that ranges between $0-60$. The scale has been shown to be valid, sensitive to change (38), and highly consistent $(\alpha=0.87-0.92$ in various German samples (32). Cronbach's $\alpha$ for this study was 0.88 .

\section{Secondary outcome measures}

General and work-specific self-efficacy. The 10-item General Self-Efficacy Scale (GSE) developed by Schwarzer and Jerusalem (39) assesses a general sense of perceived self-efficacy, with the goal of predicting the ability to cope with daily problems and adapt after experiencing stressful life events. The participant is asked to evaluate statements on a 4-point Likert-type scale $(1=$ not at all true, $2=$ hardly true, $3=$ moderately true, $4=$ completely true) (eg, "I can typically handle whatever comes my way"). A higher score indicates higher self-efficacy. The item values can be summarized to a total score that ranges between 10-40. Additionally, work-related self-efficacy (WSE) was assessed using the Teacher Self-Efficacy Scale (40). The scale assesses 10 items of perceived self-efficacy with regard to occupational performance. Participants respond to every statement using a four-point Likert-type scale ( $1=$ not at all true, $2=$ hardly true, $3=$ moderately true, $4=$ completely true) (eg, "If my lessons are disturbed, I am confident and stay calm"). A higher score indicates higher perceived work-specific self-efficacy. The item values can be summarized to a total score that ranges between 10-40. Both scales have been shown to have good reliability and validity in a number of studies (41). Cronbach's $\alpha$ was 0.85 and 0.75 for the GSE and WSE, respectively.

Burnout symptoms. The Maslach Burnout Inventory for people working in human services (MBI-D) (42) was used to assess symptoms of burnout. The total scale consists of 21 items and evaluates three types of symptoms: emotional exhaustion (EE, 9 items), depersonalization (DP, 5 items) and personal accomplishment (PA, 7 items). Participants are asked to rate statements on a 7-point Likert-type scale ( $1=$ it never happens to me, $2=\ldots$ very rarely $\ldots, 3=\ldots$ rarely $\ldots, 4=\ldots$ sometimes..., $5=\ldots$ quite often. .6 =it happens to me every day) (eg, "I feel frustrated with my job"). Higher scores on EE and DP indicate a higher risk of burnout, whereas a higher PA indicates a lower risk of burnout. The item values of each subdomain can be summarized to a single score for EE (ranges between 9-54), DP (ranges between 5-30) and PA (ranges between 7-42). The reliability and validity of this inventory are adequate (43). In this study, the EE Cronbach's $\alpha$ was 0.85 for EE, 0.75 for DP, and 0.70 for PA.

Stress. Symptoms of stress were measured with the Perceived Stress Questionnaire (PSQ) $(44,45)$. This questionnaire consists of 20 items measuring perceived stressful situations and stress reactions in four subdomains: worries, tension, joy, and demands. The respondent is asked to rate how often he or she experienced certain situations or reactions on a 4-point Likert-type scale $(1=$ almost never, $2=$ sometimes, $3=$ often, $4=$ usually) during the past four weeks. Higher scores on the subdomains of worries, tension and demands denote a higher level of perceived stress, whereas a higher score for joy denotes a lower level of perceived stress. An average score of all item scores can be calculated. After a linear transformation the overall score ranges between $0-1$. The reliability and validity of the scale are adequate (44). In this study, the Cronbach's $\alpha$ for the PSQ was 0.90 .

Worrying. The Penn State Worry Questionnaire (PSWQ) $(46,47)$ is a commonly used, self-rating measure of (pathological) worrying. The 16 items are directed at the excessiveness, duration, and uncontrollability of worry on a 7 -point Likert-type scale $(0=$ never, $1=$ very rarely, $2=$ rarely, $3=$ sometimes, $4=$ often, $5=$ very often, $6=$ almost always) for events that occurred during the past week, where a higher score indicates more worrying. The item values can be summarized to a total score, varying between $0-96$. The scale displayed good internal consistency $[\alpha=0.86$ (47)]. In this study, Cronbach's $\alpha$ for the PSWQ was 0.95 .

Health-related quality of life. Health-related quality of life was measured with the SF-12 Health Survey (48). The SF-12 consists of 12 items considering 8 health subdomains (physical functioning, physical and emotional functioning, bodily pain, general health, vitality, social functioning, and mental health) for the past four weeks of the subject's life. Some items are scored as absent/ present (eg, "During the past four weeks, have you had any of the following problems with your work or other regular daily activities as a result of any emotional problems?"), whereas others are scored on a Likert-type scale with varying ranges. The SF-12 algorithm generates two summary scores: a physical component score (PCS) and 
a mental health component score (MCS). Each score ranges from $0-100$, with a higher score indicating a higher degree of functioning. Cronbach's $\alpha$ was 0.80 and 0.68 for the physical and mental health component scores, respectively, in the present study.

Absenteeism: Absence from work due to sickness was measured as the self-reported sick leave of participants during the past four weeks (yes/no) and the self-rated amount of total days on sick leave during the past four weeks. Table 1 provides an overview of the measurement tools for all assessments.

\section{Data analysis}

All analyses were performed according to the recommendations of the CONSORT-Statement for RCT. Analyses were based on intention-to-treat (ITT) procedures.

Missing data. Baseline data on primary and secondary outcomes were provided by all participants. Absences from follow-up assessments were imputed using a Markov Chain Monte Carlo multivariate imputation algorithm (missing data module in SPSS 20) with 10 estimations per missing value. To assess potential systematic effects of non-ignorable missing data, pattern mixture analyses for multi-level longitudinal approaches (49) were conducted to test whether the intervention effect was systematically related to missing data. For this purpose, the missing-data pattern of each participant was first coded in 1 of 16 possible missing patterns $\left(2^{4 ;}\right.$ [Missing Yes/No number of assessments]. The created between-subject variable was then included in a three-way interaction (missing pattern $\times$ condition $\times$ change in symptom severity over time) in the main outcome analyses.

The assumed superiority of the iPST compared to the WLC group was tested with regard to (i) changes in depressive symptom severity and secondary outcomes from baseline (T1) to post-intervention (T2) and follow-up assessments (T3, T4), (ii) number of participants with a reliable change in depressive symptom severity, and (iii) number of participants who reached symptom-free status.

Change in depressive symptom severity and secondary outcomes. To assess the differences between conditions in terms of the degree of change in outcomes over time, mixed-effects models (MEM) of change were calculated for the primary and secondary outcome variables. In the models, time was included at level 1 (dummy-coded indicator variables for changes in symptoms between T1-T2, T1-T3, and T1-T4), treatment conditions $(0=\mathrm{WLC} ; 1=\mathrm{iPST})$ were included at level 2 (ie, participants), and all cross-level interaction effects
Table 1. Overview of the assessment instruments. [wks=weeks; mths=months; CES-D=Center For Epidemiological Studies Depression Scale; $\mathrm{MBI}=$ Maslach Burnout Inventory; $\mathrm{PSWQ=Penn}$ State Worry Questionnaire; PSQ=Perceived Stress Questionnaire; SF-12=Short Form Health Survey; TiC-P=Trimbos and Institute of Medical Technology Assessment Cost Questionnaire for Psychiatry]

\begin{tabular}{|c|c|c|c|c|c|}
\hline \multicolumn{2}{|c|}{ Instrument Aim } & \multirow{2}{*}{$\begin{array}{c}\begin{array}{c}\mathrm{T} 1 \\
\text { (baseline) }\end{array} \\
\mathrm{x}\end{array}$} & \multirow{2}{*}{$\begin{array}{c}\text { T2 } \\
(6 \text { wks })\end{array}$} & \multirow{2}{*}{$\begin{array}{c}\begin{array}{c}\text { T3 } \\
(3 \text { mths })(\end{array} \\
x\end{array}$} & \multirow{2}{*}{$\begin{array}{c}\begin{array}{c}\mathrm{T} 4 \\
(6 \mathrm{mths})\end{array} \\
x\end{array}$} \\
\hline CES-D & $\begin{array}{l}\text { Depressive } \\
\text { symptomatology }\end{array}$ & & & & \\
\hline GSE & General self-efficacy & $x$ & $x$ & $x$ & $x$ \\
\hline WSE & $\begin{array}{l}\text { Work-specific } \\
\text { self-efficacy }\end{array}$ & $x$ & $x$ & $x$ & $x$ \\
\hline $\mathrm{MBI}$ & Burnout symptoms & $x$ & $x$ & $x$ & $x$ \\
\hline PSWQ & Pathological worries & $x$ & $\mathrm{x}$ & $x$ & $x$ \\
\hline PSQ & Perceived stress & $x$ & $x$ & $x$ & $x$ \\
\hline SF-12 & Quality of life & $x$ & $x$ & $x$ & $x$ \\
\hline TiC-P & $\begin{array}{l}\text { Sickness absence from } \\
\text { work }\end{array}$ & $x$ & $\mathrm{x}$ & $\mathrm{x}$ & $\mathrm{x}$ \\
\hline $\begin{array}{l}\text { Other } \\
\text { questions }\end{array}$ & Socio-demographics & $x$ & - & - & - \\
\hline
\end{tabular}

were included (condition $\times \mathrm{T} 1-\mathrm{T} 2$; condition $\times \mathrm{T} 1-\mathrm{T} 3$; condition $\times \mathrm{T} 1-\mathrm{T} 4)$. We imposed no restrictions whatsoever on the covariance matrix; therefore, slopes indicating changes between measurement occasions were allowed to vary freely between participants. All effects were estimated using the full information maximum likelihood (FIML) procedure. T-tests were used to assess differences between groups in terms of absenteeism at T2, T3, and T4. For all outcomes, Cohen's d (50) was calculated based on the imputed dataset by standardizing the pre-post differences between groups based on the pooled standard deviation of change scores. Moreover, $95 \%$ confidence intervals $(95 \% \mathrm{CI})$ for effect sizes were calculated according to Rosnow and Rosenthal (51). Additional per protocol analyses were conducted based on the sample of participants who adequately adhered to the intervention protocol (by completing at least four out of five intervention sessions).

Reliable change: To assess improvements of the primary outcome (depressive symptom severity) on an individual level, we examined the number of participants who displayed a reliable change according to the widely used reliable change index of Jacobsen and Truax (52). Participants were defined as reliably improved if their CES-D-score declined from baseline to post-assessment with a reliable change index $>1.96$ (8.65 points in the CES-D).

Absence of subclinical depression: Because subclinical symptoms of depression are the key risk factor for developing severe depression $(53,54)$, the number of participants who were free of depressive symptoms was 
counted in both conditions. The absence of subclinical depression was predefined as a drop below the cut-off value of 16 on the CES-D.

We also calculated the number needed to treat (NNT) and $95 \% \mathrm{CI}$ that indicate the number of teachers that needed to participate to generate one additional positive outcome.

All analyses were performed with SPSS version 20 (IBM Corp, Armonk, NY, USA). All reported P-values are two-sided with a significance level of 0.05 .

\section{Results}

\section{Participants}

Figure 1 summarizes the enrollment and flow of participants throughout the study. Out of 245 individuals assessed for eligibility, 63 (25.7\%) were excluded because of a screening CES-D score of $>16$. Another 30 $(12.2 \%)$ individuals did not provide informed consent. Two individuals (1\%) withdrew from the study before

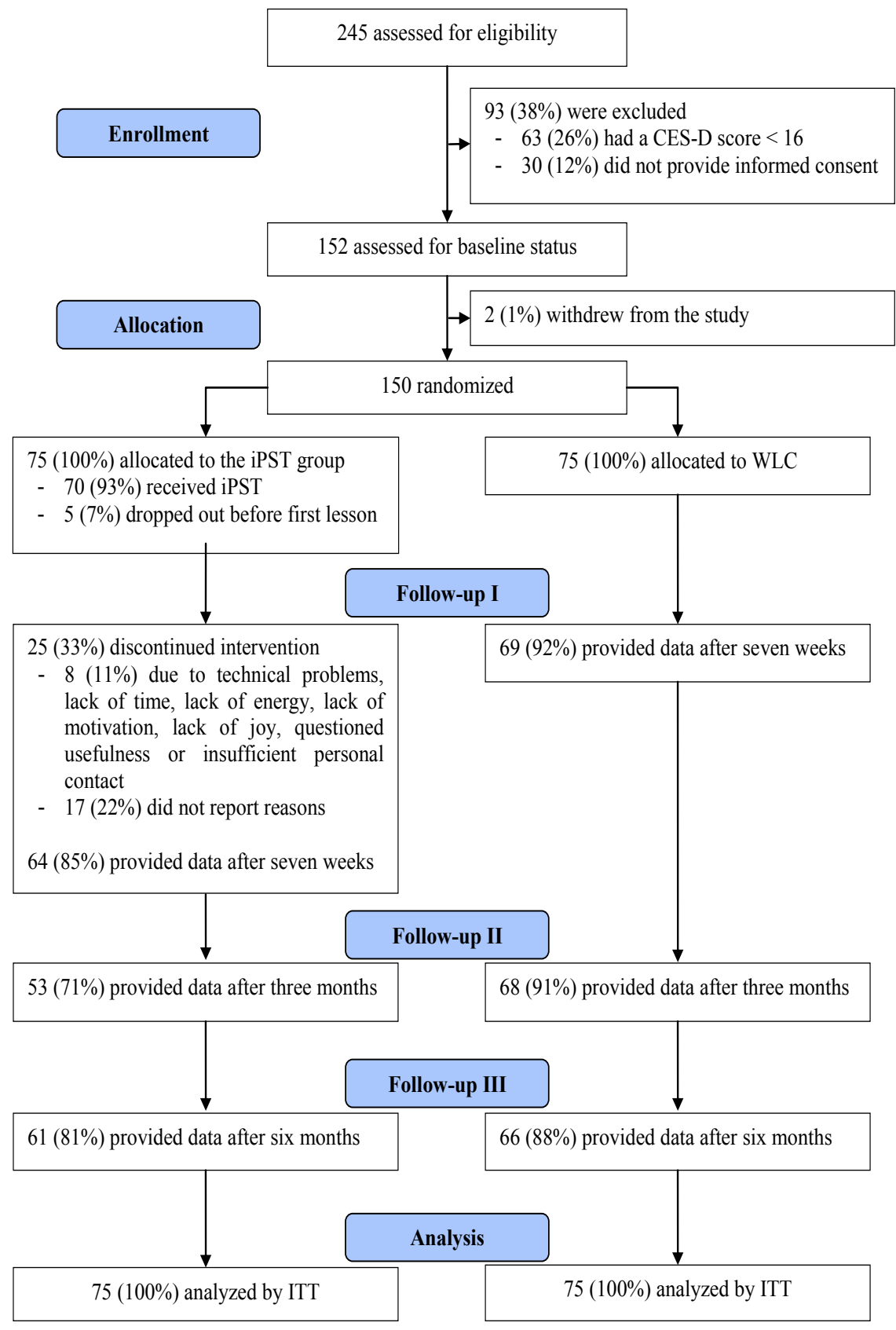

Figure 1. Flow of study participants. CES-D = Center for Epidemiologic Studies Depression Scale; iPST=internetbased guided problem-solving training; ITT=intention-to-treat; WLC=waitlist control group.] 
the baseline assessment and randomization procedure. After these exclusions, 150 (61.2\%) teachers were randomized to either the iPST or WLC group.

\section{Missing data}

There were 133 (88.7\%) participants who completed the online-based questionnaire seven weeks after randomization. There were $121(80.7 \%)$ participants who completed the three-month follow-up, and 127 (84.7\%) provided data at the six-month follow-up. The respective numbers of responses for the iPST group were 64 $(85.3 \%), 53(70.6 \%)$, and $61(81.3 \%)$ compared to 69 (92\%), $68(90.6 \%)$, and $66(88 \%)$, respectively, for the WLC group. Groups did not differ with regard to missing data (all $\mathrm{P}>0.10$ ), except for the three-month follow-up $\left(\mathrm{Chi}^{2}=9.62, \mathrm{P}<0.05\right)$. Participants who did not provide data at one of the follow-up assessments did not differ from participants without missing data on baseline depression severity scores or any other baseline characteristics, except for pathological worries $(\mathrm{P}=0.02)$. All other differences were not significant (all $\mathrm{P}>0.10$ ). Little's overall test of randomness indicated that the missing data were completely random, and thus, multiple imputations of the missing data could be conducted (55). Pattern mixture analyses did not indicate a significant interaction between the pattern of missing data and the primary outcome. Thus, missing data did not appear to bias the results.

\section{Baseline characteristics}

As indicated in table 2, the sample consisted of 150 teachers with an average age of 47.1 years [standard deviation $(\mathrm{SD})=8.2]$. The majority of participants $(\mathrm{N}=125 ; 83.3 \%)$ were female, and 93 participants
$(62.0 \%)$ were married or in a steady cohabiting relationship. The participants had an average of 19 years of teaching experience $(\mathrm{SD}=9.6)$. Only $30(24.2 \%)$ had participated in any traditional mental health training programs before the study. Whereas 55 (44.4\%) of the participants had never taken part in a mental health training program or psychotherapy, 61 (48.8\%) participants had received psychotherapy in the past. Relatively few participants reported being currently on sick leave $(\mathrm{N}=7 ; 4.7 \%)$, and the number of total sick days within the last four weeks was also comparatively low (mean 2.2, SD 5.6). Table 3 provides descriptive data for all outcome variables. No meaningful differences were found between the iPST and WLC group for any of the baseline variables, which indicates that the randomization procedure was successful.

\section{Intervention usage}

Out of 75 participants in the iPST group, 70 (93\%) completed at least one lesson, $62(83 \%)$ completed two lessons, 56 (75\%) completed three lessons, and 52 $(70 \%)$ completed four lessons in the training program. Only $45(60 \%)$ completed all five lessons of the training. Attrition was not significantly associated with any specific session $\left(\mathrm{Chi}^{2}=1.67 ; \mathrm{df}=4 ; \mathrm{P}=0.79\right)$. Qualitative analyses of problems indicated that both work and non-work problems were reported and addressed during the training. On average, participants reported $4.15(\mathrm{SD}=2.09)$ different problems; $1.46(\mathrm{SD}=1.40)$ of the problems were reported as being work-related (eg, lack of esteem from supervisor), $1.97(\mathrm{SD}=1.98)$ were related to private life (eg, arguing with own kids), and $0.72(\mathrm{SD}=1.34)$ could be assigned to both domains (eg, difficulties to relax).

Table 2. Demographic characteristics: means/counts and standard deviations/percentages before the treatment. [iPST=internet-based guided problem-solving training; WLC=waitlist control group; SD=standard deviation]

\begin{tabular}{|c|c|c|c|c|c|c|c|c|c|c|c|c|}
\hline & \multicolumn{4}{|c|}{ All } & \multicolumn{4}{|c|}{ iPST } & \multicolumn{4}{|c|}{ WLC } \\
\hline & $\mathrm{N}$ & $\%$ & Mean & SD & $\mathrm{N}$ & $\%$ & Mean & SD & $\mathrm{N}$ & $\%$ & Mean & SD \\
\hline Age $^{a}$ & & & 47.1 & 8.2 & & & 46.4 & 9.2 & & & 47.8 & 7.3 \\
\hline Years of occupational experience ${ }^{b}$ & & & 19.0 & 9.6 & & & 18.7 & 10.4 & & & 19.2 & 9.0 \\
\hline Weekly working hours & & & 42.0 & 14.6 & & & 42.4 & 16.9 & & & 41.7 & 12.1 \\
\hline Days on sick leave during the past four weeks & & & 2.2 & 5.6 & & & 2.8 & 6.5 & & & 1.6 & 4.5 \\
\hline Females & 125 & 83.3 & & & 62 & 83.3 & & & 63 & 84.0 & & \\
\hline Married/partnership a & 93 & 74.4 & & & 44 & 75.9 & & & 49 & 73.1 & & \\
\hline $\begin{array}{l}\text { Experiences with occupational mental health } \\
\text { training c }\end{array}$ & 30 & 24.2 & & & 17 & 29.3 & & & 13 & 19.7 & & \\
\hline Experiences with psychotherapy ${ }^{c}$ & 61 & 48.8 & & & 26 & 44.8 & & & 35 & 52.1 & & \\
\hline
\end{tabular}

a Due to missing data, some means/counts refer to a subsample with $\mathrm{N}=125$.

${ }^{b}$ Due to missing data, some means/counts refer to a subsample with $\mathrm{N}=120$.

c Due to missing data, some means/counts refer to a subsample with $\mathrm{N}=124$. 
Table 3. Means and standard deviations of outcome variables at baseline, post-treatment, and at three-month and six-month follow-ups (intention-to-treat sample). [iPST=internet-based guided problem-solving training; WLC=waitlist control group; SD=standard deviation; CES-D=Center For Epidemiological Studies Depression Scale; GSE=General Self-Efficacy Scale; WSE=Work-specific Self-Efficacy Scale; $\mathrm{MBI}=$ Maslach Burnout Inventory; $\mathrm{EE}=$ =motional exhaustion; $\mathrm{DP}=$ depersonalization; $\mathrm{PA}=$ personal accomplishment; $\mathrm{PSWQ=Penn} \mathrm{State}$ Worry Questionnaire; PSQ=Perceived Stress Questionnaire; SF-12-PCS=Short Form Health Survey - physical component summary; MCS=mental component summary]

\begin{tabular}{|c|c|c|c|c|c|c|c|c|c|c|c|c|c|c|c|c|}
\hline \multirow[t]{3}{*}{ Outcome } & \multicolumn{4}{|c|}{$\mathrm{T} 1$} & \multicolumn{4}{|c|}{ T2 } & \multicolumn{4}{|c|}{ T3 } & \multicolumn{4}{|c|}{ T4 } \\
\hline & \multicolumn{2}{|c|}{ iPST } & \multicolumn{2}{|c|}{ WLC } & \multicolumn{2}{|c|}{ iPST } & \multicolumn{2}{|c|}{ WLC } & \multicolumn{2}{|c|}{ iPST } & \multicolumn{2}{|c|}{ WLC } & \multicolumn{2}{|c|}{ iPST } & \multicolumn{2}{|c|}{ WLC } \\
\hline & Mean & SD & Mean & SD & Mean & SD & Mean & SD & Mean & SD & Mean & SD & Mean & SD & Mean & SD \\
\hline CES-D & 22.76 & 9.24 & 2.81 & 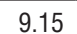 & 37 & 8.43 & 21.20 & & .53 & 13.80 & 19.87 & 14.03 & 5.87 & 10.07 & 19.91 & 10.42 \\
\hline GSE & 25.17 & 4.64 & 25.09 & & 8 & 4.03 & 25.98 & & 16 & 6.02 & 26.14 & & .43 & 9 & 39 & 4.69 \\
\hline WSE & 26.95 & 4.32 & 27.43 & 3.98 & 57 & 4.78 & 26.79 & 4.12 & 29.41 & 5.11 & 27.79 & 45 & 3.63 & 37 & 27.45 & 62 \\
\hline & 38.39 & 7.39 & 38.85 & 6. & 36 & 7.82 & 38.14 & & 33.75 & 10.65 & 38 & 8.22 & & 9.84 & 38.01 & .52 \\
\hline & 12.80 & 4.71 & 11.99 & 4.10 & 11.80 & 4.54 & 12.03 & 4. & 11.91 & 4.46 & 11.71 & 4.47 & 11.34 & 4.40 & 11.96 & 4.37 \\
\hline MBI- & 30.99 & 3.8 & 30.91 & 3.38 & 31.97 & 4.02 & 30.97 & 3.41 & 31.96 & 4.70 & 31.00 & 3.50 & 32.52 & 5.06 & 31.24 & 4.16 \\
\hline PSWO & 58.49 & 16.30 & 55.90 & 15.70 & 42.86 & 23.01 & 53.74 & 19.40 & 33.66 & 27.54 & 47.57 & 24.46 & 39.45 & 26.88 & 50.13 & 21.37 \\
\hline PSQ & 0.66 & 0.1 & 0.67 & 0.14 & 0.55 & 0.19 & 0.62 & 0.17 & 0.48 & 0.27 & 0.56 & 0.25 & 0.53 & 0.19 & 0.60 & 0.16 \\
\hline SF12-PCS & 44.25 & 10.81 & 46.76 & 8.76 & 45.48 & 13.97 & 45.78 & 10.50 & 46.55 & 18.47 & 46.08 & 11.25 & 47.29 & 11.37 & 45.36 & 9.59 \\
\hline SF12-MCS & 34.01 & 8.62 & 34.25 & 9.51 & 40.48 & 13.45 & 35.72 & 10.20 & 44.03 & 21.36 & 37.80 & 13.90 & 42.59 & 11.83 & 39.55 & 10.35 \\
\hline Absenteeisma & 2.80 & 6.45 & 1.61 & 4.50 & 1.56 & 5.36 & 1.68 & 4.16 & 1.86 & 6.05 & 1.70 & 5.21 & 1.18 & 4.27 & 2.76 & 7.75 \\
\hline
\end{tabular}

a Total days on sick leave during the past four weeks.

Primary outcome analyses - changes in depressive symptom severity

Figure 2 displays the estimated changes in the primary outcome (CES-D) over time based on the ITT MEM analyses. The first row in table $4 \mathrm{a}$ lists the results of the MEM analyses in detail. The intercept represents the estimated initial level of depressive symptoms before treatment (T1) in the WLC group. The regression coefficient $\mathrm{T} 1-\mathrm{T} 2$ represents an estimator for change in depressive symptoms between the baseline and seven-week post-treatment assessment in the WLC group. The regression coefficients $\mathrm{T} 1-\mathrm{T} 3$ and

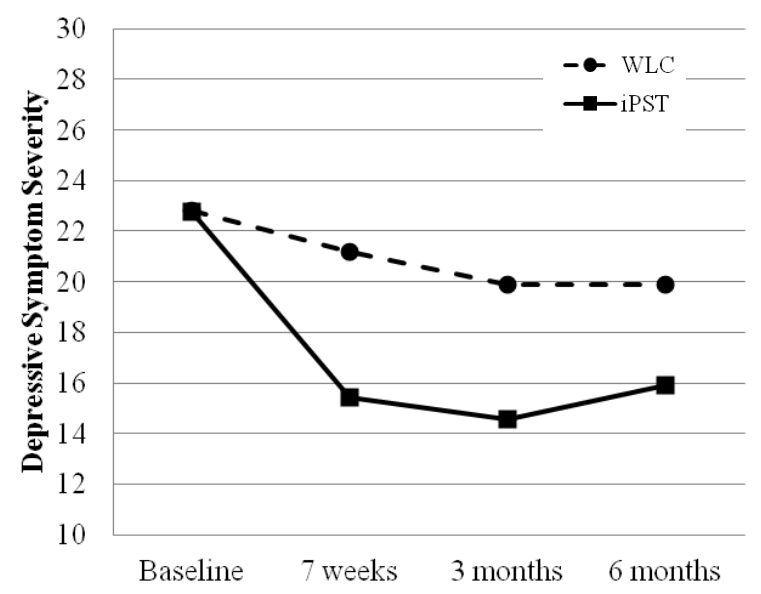

Figure 2. Estimated course of depressive symptom severity over time (based on the mixed-effect model).
T1-T4 represent estimates for changes between the baseline and follow-up assessments after three and six months, respectively, in the WLC group. The regression coefficient for "group" represents the estimated difference between the WLC and iPST groups before treatment. The regression coefficients $\mathrm{T} 1-\mathrm{T} 2 \times$ group, $\mathrm{T} 1-\mathrm{T} 3 \times$ group, and $\mathrm{T} 1-\mathrm{T} 4 \times$ group are estimates of cross-level interaction effects. These coefficients represent estimates of the group differences in "change from baseline to post-treatment", for the three- and six-month follow-ups, respectively.

There was no significant effect on T1-T2 and T1$\mathrm{T}$, indicating no significant change from baseline to post-intervention and three-month follow-up in the WLC group. The significant effect on T1-T4 indicates a small decrease in the severity of depressive symptoms over time. The significant negative interaction effect for $\mathrm{T} 1-\mathrm{T} 2 \times$ group indicates a greater decrease in depressive symptom severity in the iPST compared to the WLC group. Moreover, the larger decrease in the iPST group was maintained at both follow-ups (as indicated by significant negative interaction effects for $\mathrm{T} 1-\mathrm{T} 3 \times$ group and T1-T4×group), although the interaction effects declined slightly over time. Effect sizes were medium to large for the differences between iPST and WLC in "change from baseline to post-treatment", and the effects were small to moderate for "change from baseline to three- and six-month follow-up" (see table 5).

A separate analysis examining the intervention effects for the per protocol subsample (participants who adhered to at least four training lessons; $\mathrm{N}=52$ ) was conducted. This per protocol analysis revealed larger effects compared to the ITT sample for changes from baseline to post treatment $(\mathrm{d}=0.90,95 \%$ CI $0.53-1.27$ ), 
to the three-month ( $\mathrm{d}=0.51,95 \% \mathrm{CI} 0.16-0.87)$ and sixmonth ( $\mathrm{d}=0.58,95 \% \mathrm{CI} 0.22-0.94)$ follow-up.

\section{Reliable and significant change in depressive symptom severity}

The iPST group was superior to the WLC group in terms of the number of participants with reliable improvements in depressive symptom severity from before the treatment to (i) seven weeks after the treatment (iPST: $\mathrm{N}=37$ [49.3\%], WLC: $\mathrm{N}=16$ [21.3\%], $\mathrm{P}<0.01$ ), (ii) three months after the treatment (iPST: $\mathrm{N}=35$ [46.7\%], WLC: $\mathrm{N}=25[33.3 \%], \mathrm{P}=0.10$ ), and (iii) six months after the treatment (iPST: $\mathrm{N}=34$ [45.3\%], WLC: $\mathrm{N}=17$ [22.7\%], $\mathrm{P}<0.01)$. The NNT to achieve one reliable improvement from baseline to post treatment was 3.5 (95\% CI 2.3-6.4). This result indicates three teachers had to participate in the intervention iPST to result in one more teacher having reliable improvement in depressive symptom severity compared to the WLC group. At the three- and six-month follow-ups, the NNT values were 7.5 (95\% CI 3.4-10.6) and 4.4 (95\% CI 2.6-12.5), respectively.

Further analyses indicated that significantly more participants in the iPST group (N=44, 58.7\%) reached symptom-free status $(\mathrm{CES}-\mathrm{D}<16)$ seven weeks after randomization compared to the WLC group $(\mathrm{N}=16$, $21.3 \%)(\mathrm{NNT}=2.6 ; 95 \%$ CI 1.9-4.3, $\mathrm{P}<0.01)$. After three months, $45(60 \%)$ teachers in the iPST group and 31 $(41.3 \%)$ teachers in the WLC group reached symptomfree status (NNT $=5.3 ; 95 \%$ CI 2.9-33.9, $\mathrm{P}=0.02$ ). After six months, $38(50.7 \%)$ teachers in the iPST group and $27(36 \%)$ in the WLC group (NNT=6.8; 95\% CI
3.2-10.6, $\mathrm{P}=0.07$ ) had passed the cut-off point on the CES-D and thus reached symptom-free status.

\section{Secondary outcomes}

Table 4 presents the results of the ITT MEM analyses for changes in secondary outcomes. Significant cross-level interaction effects indicated that there were betweengroup differences in change from baseline to posttreatment (T1-T2×group) that favored the iPST group for all outcomes, except for the MBI scales (EE: $\mathrm{P}=0.13$; PA: $\mathrm{P}=0.13$; DP: $\mathrm{P}=0.15$ ) and the physical health scale of the SF-12 ( $\mathrm{P}=0.21)$. Effect sizes for secondary outcomes with significant interactions ranged from $\mathrm{d}=0.36$ (95\% CI 0.04-0.69) for perceived stress to $\mathrm{d}=0.63(95 \%$ CI 0.30-0.96) for worrying (table 5). Moreover, the significant cross-level interaction effects $\mathrm{T} 1-\mathrm{T} 3 \times$ group and T1-T4×group indicate that between-group differences from baseline to the three- and six-month follow-ups favor the iPST group for of the majority of outcomes. Interactions were not significant for changes from baseline to the three-month follow-up in the MBI subdomains of DP $(\mathrm{P}=0.43)$ and $\mathrm{PA}(\mathrm{P}=0.22)$, and a trend toward significance was observed for perceived stress $(\mathrm{P}=0.09)$. Changes from baseline to six-month follow-up were only non-significant for the MBI scale of $\mathrm{PA}(\mathrm{P}=0.22)$. Effect sizes of significant interactions were moderate, ranging from $\mathrm{d}=0.38(95 \% \mathrm{CI} 0.06-0.70)$ for GSE to $d=0.62(95 \%$ CI $0.29-0.95)$ for worrying at the three-month follow-up. At the six-month follow-up, the effect sizes of significant interactions were small to moderate, ranging from $\mathrm{d}=0.33(95 \% \mathrm{CI} 0.01-0.65)$ for DP to $\mathrm{d}=0.54$ (95\% CI $0.21-0.68)$ for worrying.

Table 4a. Differences in changes of primary and secondary outcomes over time in the mixed-effects models (intention-to-treat sample). [iPST=internet-based guided problem-solving training; WLC=waitlist control group; SE=standard error; CES-D=Center For Epidemiological Studies Depression Scale; GSE=General Self-Efficacy Scale; WSE=Work-specific Self-Efficacy Scale; MBI=Maslach Burnout Inventory; $\mathrm{EE}=$ =motional exhaustion; $\mathrm{DP}=$ depersonalization; $\mathrm{PA}=$ personal accomplishment; $P S W Q=P$ enn State Worry Questionnaire; $P S Q=P e r c e i v e d$ Stress Questionnaire; SF-12=Short Form Health Survey; PCS=physical component summary; MCS=mental component summary]

\begin{tabular}{|c|c|c|c|c|c|c|c|c|c|c|}
\hline \multirow[t]{3}{*}{ Outcome } & \multicolumn{8}{|c|}{ WLC over time } & \multirow{2}{*}{\multicolumn{2}{|c|}{$\begin{array}{c}\text { iPST versus WLC } \\
\text { Group }\end{array}$}} \\
\hline & \multicolumn{2}{|c|}{ Intercept T1 } & \multicolumn{2}{|c|}{$\mathrm{T} 1-\mathrm{T} 2$} & \multicolumn{2}{|c|}{ T1-T3 } & \multicolumn{2}{|c|}{ T1-T4 } & & \\
\hline & $B$ & SE & $B$ & SE & $B$ & SE & $B$ & SE & $B$ & SE \\
\hline CES-D & 22.81 & 1.05 & -1.61 & 1.11 & $-2.94^{d}$ & 1.56 & $-2.90^{a}$ & 1.23 & -0.05 & 1.49 \\
\hline GSE & 25.09 & 0.52 & $0.90^{c}$ & 0.45 & $1.05 c$ & 0.52 & $1.31^{c}$ & 0.54 & 0.08 & 0.74 \\
\hline WSE & 27.43 & 0.47 & -0.65 & 0.44 & 0.35 & 0.50 & 0.02 & 0.46 & -0.48 & 0.67 \\
\hline MBI-EE & 38.89 & 0.80 & -0.75 & 0.69 & -0.26 & 0.82 & -0.88 & 0.89 & -0.50 & 1.13 \\
\hline MBI-DP & 12.03 & 0.51 & 0.00 & 0.47 & -0.32 & 0.52 & -0.07 & 0.46 & 0.77 & 0.72 \\
\hline MBI-PA & 30.89 & 0.42 & 0.07 & 0.40 & 0.11 & 0.46 & 0.35 & 0.44 & 0.09 & 0.59 \\
\hline PSWQ & 56.19 & 1.84 & -2.45 & 2.23 & $-8.62 b$ & 2.79 & $-6.06 c$ & 2.60 & 2.30 & 2.60 \\
\hline$P S Q$ & 0.67 & 0.02 & $-0.04^{c}$ & 0.02 & $-0.11^{a}$ & 0.03 & $-0.07^{b}$ & 0.02 & -0.01 & 0.02 \\
\hline SF12-PCS & 46.76 & 1.13 & -0.98 & 1.12 & -0.68 & 1.35 & -1.40 & 1.18 & -2.51 & 1.60 \\
\hline SF12-MCS & 34.35 & 1.04 & 1.37 & 1.33 & $3.45^{c}$ & 1.60 & $5.20^{a}$ & 1.39 & -0.34 & 1.47 \\
\hline
\end{tabular}

a $P<0.001$

b $P<0.01$

c $P<0.05$

d $P<0.10$ 
Table 4b.

\begin{tabular}{|c|c|c|c|c|c|c|c|c|c|}
\hline \multirow[t]{3}{*}{ Outcome } & \multicolumn{9}{|c|}{ iPST versus WLC over time } \\
\hline & \multicolumn{3}{|c|}{ T1-T2×Group } & \multicolumn{3}{|c|}{ T1-T3×Group } & \multicolumn{3}{|c|}{ T1-T4×Group } \\
\hline & $\mathrm{B}$ & SE & $95 \% \mathrm{Cl}$ & $\mathrm{B}$ & SE & $95 \% \mathrm{Cl}$ & B & SE & $95 \% \mathrm{Cl}$ \\
\hline CES-D & $-5.78^{a}$ & 1.59 & $-8.89--2.66$ & $-5.29 c$ & 2.34 & $-9.92--0.66$ & $-3.98 c$ & 1.67 & $-7.25--0.70$ \\
\hline GSE & $1.91^{b}$ & 0.65 & $0.64-3.18$ & $1.93^{c}$ & 0.74 & $0.46-3.40$ & $1.95^{c}$ & 0.77 & $0.44-3.46$ \\
\hline WSE & $2.27^{\mathrm{a}}$ & 0.63 & $1.03-3.52$ & $2.11^{b}$ & 0.71 & $0.70-3.52$ & $1.67^{c}$ & 0.69 & $0.30-3.04$ \\
\hline MBI-EE & -1.54 & 1.02 & $-3.55-0.48$ & $-4.38^{a}$ & 1.22 & $-6.79--1.97$ & $-3.00^{c}$ & 1.31 & $-5.59--0.42$ \\
\hline MBI-DP & -1.00 & 0.69 & $-2.35-0.35$ & -0.57 & 0.71 & $-1.96-0.83$ & $-1.39 c$ & 0.67 & $-2.70-0.08$ \\
\hline MBI-PA & 0.90 & 0.59 & $-0.26-2.07$ & 0.87 & 0.71 & $-0.54-2.27$ & $1.19^{d}$ & 0.65 & $-0.09-2.46$ \\
\hline PSWQ & $-13.18^{a}$ & 3.51 & $-20.13--6.23$ & $-16.21^{a}$ & 4.12 & $-24.34--8.09$ & $-12.99 \mathrm{~b}$ & 3.70 & $-20.27--8.09$ \\
\hline$P S Q$ & $-0.07 c$ & 0.03 & $-0.13--0.01$ & $-0.07^{d}$ & 0.04 & $-0.15-0.01$ & $-0.06^{c}$ & 0.03 & $-0.12--0.01$ \\
\hline SF12-PCS & 2.22 & 1.74 & $-1.23-5.67$ & 2.99 & 2.22 & $-1.44-7.41$ & $4.44^{b}$ & 1.78 & $0.94-7.94$ \\
\hline SF12-MCS & $5.11^{c}$ & 2.01 & $1.16-9.06$ & $6.57^{c}$ & 3.03 & $0.37-12.77$ & $3.38^{d}$ & 1.99 & $-0.53-7.28$ \\
\hline
\end{tabular}

a $P<0.001$

b $P<0.01$

c $P<0.05$

d $P<0.10$

Table 5. Between-group effect sizes of primary and secondary outcomes for changes from baseline to post and follow-up assessments (intention-to-treat sample). [iPST=internet-based guided problemsolving training; WLC=waitlist control group; $95 \% \mathrm{Cl}=95 \%$ confidence interval; CES-D=Center For Epidemiological Studies Depression Scale; GSE=General Self-Efficacy Scale; WSE=Work-specific Self-Efficacy Scale; $\mathrm{MBI}=$ Maslach Burnout Inventory; $\mathrm{EE}=$ =motional exhaustion; DP=depersonalization; $P A=$ personal accomplishment; $P S W Q=P e n n$ State Worry Questionnaire; $P S Q=P e r c e i v e d$ Stress Questionnaire; SF-12=Short Form Health Survey; PCS=physical component summary; MCS=mental component summary]

\begin{tabular}{|c|c|c|c|c|c|c|}
\hline \multirow[t]{2}{*}{ Outcome } & \multicolumn{2}{|c|}{$\mathrm{T} 1-\mathrm{T} 2$} & \multicolumn{2}{|r|}{ T1-T3 } & \multicolumn{2}{|r|}{$\mathrm{T} 1-\mathrm{T} 4$} \\
\hline & $d$ & $95 \% \mathrm{Cl}$ & $d$ & $95 \% \mathrm{Cl}$ & $d$ & $95 \% \mathrm{Cl}$ \\
\hline CES-D & 0.59 & $0.26-0.92$ & 0.37 & $0.05-0.70$ & 0.38 & $0.05-0.70$ \\
\hline GSE & 0.47 & 0.1 & 0.38 & & 0.40 & \\
\hline WSE & 0.56 & 0.23 & 0.47 & 0.1 & 0.40 & 0.0 \\
\hline MBI-EE & 0.24 & -0.08 & 0.54 & 0.22 & 0.38 & 0.0 \\
\hline BI-DP & 0.23 & -0.09 & 0.12 & -0.20 & 0.33 & 0.0 \\
\hline MBI-PA & 0.25 & -0.08 & 0.21 & -0.11 & 0.29 & $-0.04-0.61$ \\
\hline PSWQ & 0.63 & & 0.62 & 0.29 & 0.54 & $0.21-0.86$ \\
\hline PSQ & 0.36 & 0.04 & 0.28 & -0.05 & 0.36 & 0.03 \\
\hline SF12- & 0.19 & -0.12 & 0.19 & -0.13 & 0.39 & $0.08-0.72$ \\
\hline SF12-MCS & 0.40 & $0.09-0.73$ & 0.35 & $0.03-0.68$ & 0.27 & $-0.05-0.59$ \\
\hline Absent ${ }^{a}$ & 0.22 & $-0.10-0.54$ & 0.14 & $-0.18-0.46$ & 0.37 & $0.04-0.69$ \\
\hline
\end{tabular}

a Total days on sick leave during the past four weeks.

Absenteeism. Only 19 participants (12.7\%) reported having been absent at least one day on sick leave within the past four weeks prior post-treatment assessment, (iPST $=7$ [4.7\%], WLC=12 [8\%], $\mathrm{P}=0.62$ ), and only 19 participants $(12.7 \%)$ reported sick leave leading up to the three-month follow-up (iPST $=6$ [4\%], WLC $=13$ [8.7\%], $\mathrm{P}=0.40)$. At the six-month follow-up, 29 (19.3\%) participants reported absence from work (iPST $=11$ [7.3\%], $\mathrm{WLC}=18$ [12\%], $\mathrm{P}=0.26$ ). The results were all in favor of the iPST group but did not reach the level of statistical significance. Total days on sick leave from work were also very low (see table 5), with no significant differences between the iPST and WLC groups when assessed immediately after treatment $\left(\mathrm{t}_{(148)}=0.15, \mathrm{P}=0.88\right)$, at the three-month follow-up $\left(\mathrm{t}_{(148)}=-0.16, \mathrm{P}=0.87\right)$, or at the six-month follow-up $\left(\mathrm{t}_{(148)}=1.51, \mathrm{P}=0.13\right)$.

\section{Discussion}

The present study aimed to evaluate the efficacy of iPST for teachers in a two-armed RCT. The results indicated that after seven weeks, three months and six months, the iPST group displayed a significantly greater reduction in depressive symptom severity. Furthermore, significantly more participants in the iPST group displayed a reliable change in depressive symptom severity and achieved symptom-free status. The iPST group also displayed greater improvements in secondary outcomes (workspecific and general self-efficacy, perceived stress, quality of life, pathological worrying) than the WLC group. No significant effects were found for the burnout dimension of PA or absenteeism.

To the best of our knowledge, this study is the first to evaluate a worker-directed internet-based guided selfhelp intervention aimed at reducing depressive symptoms among teachers. For approximately $80 \%$ of study participants, it was their first time taking part in mental health training, which indicates that internet-based interventions can attract participants who do not make use of available mental health programs. Moreover, adherence to the program was good: $70 \%$ of the iPST participants completed at least four of the five training lessons. The results of this study demonstrate that even 
a short intervention can produce substantial and enduring effects. Qualitative analyses of participant problems indicated that problems from both the work and nonwork domains were reported and addressed during the intervention. This result supports findings emphasizing the importance of non-work determinants for the prediction of a worker's mental health (56).

The changes in depressive symptoms correspond to results from earlier studies of worker-directed interventions, which had effect sizes of $\mathrm{d}=0.13$ (24), $\mathrm{d}=0.20$ (23), $\mathrm{d}=0.40$ (12), and $\mathrm{d}=0.82$ (22) when targeting employees with heightened levels of occupational stress. Only a few earlier studies have investigated the intermediate-term effects of occupational interventions; for instance, Grime (22) reported $\mathrm{d}=0.60$ after three months and $\mathrm{d}=0.30$ after six months. In the present study, the positive effects on depressive symptoms and stress-related outcomes (eg, worrying, work-related and general self-efficacy) were moderate to high in the medium term (after three or six months). The effects of iPST on depressive symptoms exceeded the relatively small effects of traditional occupational health interventions reported in meta-analyses $[\mathrm{d}=0.28(25)$ or $\mathrm{d}=0.33$ (27)]. In this study, an effect size of $d=0.59$ with regard to the reduction in depressive symptoms from baseline to seven weeks after treatment was observed, which is slightly higher than the reduction observed in other occupational health interventions. It could be that participants with a high level of education display greater responses, and the sample of teachers may have been biased towards individuals with a high level of education. Some studies have found that a high education level is associated with better treatment outcomes in internet-based interventions $(57,58)$. Other studies (59-61) did not find such an association. Thus, future research should attempt to clarify how education influences treatment outcomes in internet-based selfhelp interventions.

Our results are also in agreement with studies that evaluated non-worker-directed internet-based self-help interventions for depressive symptoms. For example, in a recent meta-analysis of RCT of computer and internetbased interventions for depressive symptoms, Richard and Richardson (17) found a mean effect size of $d=0.56$. The results of this study also agree with those of studies evaluating the original Dutch version of iPST in the general population, which had a post-intervention effect size on depressive symptoms of $\mathrm{d}=0.50$ (35) and $\mathrm{d}=0.47$ (36).

The present study found significant effects on secondary outcomes that are explicitly related to the occupational context. Compared to the WLC group, the iPST group displayed greater improvements in work-specific self-efficacy and the burnout dimension of DP, with small to moderate effects, as well as moderate effects on the burnout dimension of EE. No improvements were observed for absenteeism. One possible reason for this lack of improvement for absenteeism may be that the number of participants on sick leave and the reported number of sick days were small for all time points [eg, for the six-month follow-up: iPST=1.18 (4.27); $\mathrm{WLC}=2.76(7.75)]$.

As in many other internet-based interventions focusing on depression and health behavior, women were overrepresented in this trial (83.3\%), even when considering the actual proportion of female teachers in Germany $(70.9 \%)(62)$. Future studies should replicate the results in samples with a higher proportion of male participants.

\section{Study limitations}

The results should be viewed in the context of the study population which was comprised of a sample of highly educated school teachers. The findings may only be valid for populations with comparable demographic and job-related characteristics (high job security, relatively high income, and a high level of job autonomy); the findings may not be applicable to other types of occupations. In addition, the assessments relied exclusively on self-reported impairment at particular time points. Future studies should also include independent outcome evaluation, such as observer-based depression measures or biological outcome indicators. We did not assess treatment-as-usual utilization (eg, psychological or pharmacological co-treatment) of participants during the study period. Thus, we were not able to control for co-treatment in the analysis and cannot rule out the possibility that results are biased. The potential bias could result in an over- or underestimation of the true treatment effect.

It is often assumed that internet-based self-help interventions offer a more cost-effective treatment alternative than traditional face-to-face interventions because they typically require fewer resources (eg, travel time and costs are eliminated for both participants and trainers through the use of self-help materials). However, this study did not measure all necessary costs to conduct proper cost-effectiveness analyses (eg, health-care utilization, presenteeism). Thus, future studies are needed to clarify whether providing low-threshold guided self-help interventions to employees with depressive symptoms indeed offers a good return on investment. Future trials should replicate our findings in other working populations, particularly in populations that include a higher proportion of men or individuals of low socioeconomic status. Future studies should also explore different ways to improve the current intervention in future trials. For example, the current trial only included limited interactive elements, such as videos, diaries and persuasive design elements. A recent systematic review demonstrated that such persuasive elements are associated with higher adherence and may increase the effectiveness 
of an intervention (63). Another possible improvement would be the implementation of an emotion-regulation skill module. At work, people often must cope with difficult situations and solve problems while also addressing the challenging emotions that arise in those situations. Because deficits in the ability to adaptively cope with difficult emotions are related to various mental health problems (64), the use of effective emotion-regulation techniques (65) in low-threshold internet-based stressmanagement training appears promising $(66,67)$.

Not all employees may benefit from this particular intervention delivery to the same extent (eg, participants with low internet literacy or with only mild depressive symptoms may not benefit greatly). A recent study found that mild depressive symptoms were associated with lower effect sizes in low-intensity interventions when compared to effects for individuals with at least moderate symptoms (68). Moderator analyses should thus clarify which subgroups of participants benefit the most and the extent to which they benefit from iPST. Future studies should also investigate whether a more targeted approach, based on either the work situation of the employee (eg, cognitive and emotional task demands) or individual risk factors (eg, high effort-reward imbalance), would further improve intervention efficacy. Moreover, future studies should explore potential negative effects of internet-based occupational mental health interventions $(69,70)$.

Internet-based problem solving was effective in reducing depressive symptoms and other relevant aspects of occupational mental health among teachers. Thus, this study demonstrates that strategies and techniques typically used in traditional occupational health and stress management programs can be successfully adapted to internet-based occupational health programs. Internet-based interventions appear to have several benefits for working populations, particularly for individuals whose occupations would otherwise make interventions costly in terms of time and personal resources. Such interventions are easily scalable, and thus, only a small increase in resources is required to reach a greater proportion of the eligible population. If disseminated on a large scale, such interventions could help to reduce the burden of stress-related health problems for many employees.

\section{Acknowledgments}

The European Union funded this study (EU EFRE: ZW6-80119999, CCI 2007DE161PR001). We would like to Claudia Buntrock, Stephanie Nobis, Jo Annika Reins, and Torsten Tarnowski for providing guidance to the participants. We would further like to thank all participating associations of school psychologists and educational institutes for teacher training, as well as all employees who participated in this study.

The authors declare no conflicts of interest.

Trial Registration ISRCTN15635876.

\section{References}

1. Stansfeld S, Candy B. Psychosocial work environment and mental health - a meta-analytic review. Scand J Work Environ Health. 2006;32:443-62. http://dx.doi.org/10.5271/ sjweh. 1050

2. de Lange AH, Kompier MA, Taris TW, Geurts SA, Beckers DG, Houtman IL et al. A hard day's night: a longitudinal study on the relationships among job demands and job control, sleep quality and fatigue. J Sleep Res. 2009;18:374-83. http:// dx.doi.org/10.1111/j.1365-2869.2009.00735.x

3. Weber A, Weltle D, Lederer P. Ill health and early retirement among school principals in Bavaria. Int Arch Occup Environ Health. 2005;78:325-331. http://dx.doi.org/10.1007/s00420004-0555-9

4. Mathers CD, Loncar D. Projections of Global Mortality and Burden of Disease from 2002 to 2030. PLoS Med. 2006;3:e442. http://dx.doi.org/10.1371/journal.pmed.0030442

5. Wittchen HU, Jacobi F, Rehm J, Gustavsson A, Svensson $\mathrm{M}$, Jonsson B et al. The size and burden of mental disorders and other disorders of the brain in Europe 2010. Eur Neuropsychopharmacol. 2011;21:655-79. http://dx.doi. org/10.1016/j.euroneuro.2011.07.018

6. Andrea H, Bultmann U, Beurskens AJHM, Swaen GMH, van Schayck CP, Kant IJ. Anxiety and depression in the working population using the HAD Scale-psychometrics, prevalence and relationships with psychosocial work characteristics. Soc Psychiatry Psychiatr Epidemiol. 2004;39:637-46. http:// dx.doi.org/10.1007/s00127-004-0797-6

7. Shields M. Stress and depression in the employed population. Health Rep. 2006;17:11-29.

8. Wang J, Schmitz N, Smailes E, Sareen J, Patten S. Workplace characteristics, depression, and health-related presenteeism in a general population sample. J Occup Environ Med. 2010;52:836-42. http://dx.doi.org/10.1097/ JOM.0b013e3181ed3d80

9. Lerner D, Henke RM. What does research tell us about depression, job performance, and work productivity? J Occup Environ Med. 2008;50:401-10. http://dx.doi.org/10.1097/ JOM.0b013e31816bae50

10. Haslam C, Atkinson S, Brown SS, Haslam RA. Anxiety and depression in the workplace: effects on the individual and organisation (a focus group investigation). J Affect Disord. 2005;88:209-15. http://dx.doi.org/10.1016/j. jad.2005.07.009

11. Zetterqvist K, Maanmies J, Strom L, Andersson G. Randomized controlled trial of internet-based stress 
management. Cogn Behav Ther. 2003;32:151-60. http:// dx.doi.org/10.1080/16506070302316

12. Ruwaard J, Lange A, Bouwman M, Broeksteeg J, Schrieken B. E'-Mailed Standardized Cognitive Behavioural Treatment of Work-Related Stress: A Randomized Controlled Trial. Cogn Behav Ther. 2007;36:179-92. http://dx.doi. org/10.1080/16506070701381863

13. Mu-oz RF, Cuijpers P, Smit F, Barrera AZ, Leykin Y. Prevention of Major Depression. Annu Rev. Clin. Psychol. 2010;6:181-212. http://dx.doi.org/10.1146/annurevclinpsy-033109-132040

14. Cuijpers P, van Straten A, Warmerdam L, van Rooy, M. J. Recruiting participants for interventions to prevent the onset of depressive disorders: possible ways to increase participation rates. BMC Health Serv Res. 2010;10:181. http://dx.doi org/10.1186/1472-6963-10-181

15. Johansson R, Andersson G. Internet-based psychological treatments for depression. Expert Rev Neurotherapeutics. 2012;12:861-70. http://dx.doi.org/10.1586/ern.12.63

16. Hedman E, Ljótsson B, Lindefors N. Cognitive behavior therapy via the Internet: a systematic review of applications, clinical efficacy and cost-effectiveness. Expert Rev Pharmacoeconomics Outcomes Res. 2012;12:745-64. http:// dx.doi.org/10.1586/erp.12.67

17. Richards D, Richardson T. Computer-based psychological treatments for depression: A systematic review and metaanalysis. Clin Psychol Rev. 2012;32:329-42. http://dx.doi org/10.1016/j.cpr.2012.02.004

18. Cuijpers P, Marks IM, van Straten A, Cavanagh K, Gega L, Andersson G. Computer-aided psychotherapy for anxiety disorders: a meta-analytic review. Cogn Behav Ther. 2009;38:6682. http://dx.doi.org/10.1080/16506070802694776

19. Cheng SK, Dizon J. Computerised Cognitive Behavioural Therapy for Insomnia: A Systematic Review and MetaAnalysis. Psychother Psychosom. 2012;81:206-16. http:// dx.doi.org/10.1159/000335379

20. Riper H, Blankers M, Hadiwijaya H, Cunningham J, Clarke $\mathrm{S}$, Wiers R, et al. Effectiveness of Guided and Unguided LowIntensity Internet Interventions for Adult Alcohol Misuse: A Meta-Analysis. PloS One. 2014; 9(6), e99912. doi:10.1371/ journal.pone.0099912 http://dx.doi.org/10.1371/journal. pone.0099912

21. Civljak M, Stead LF, Hartmann-Boyce J, Sheikh A, Car J Internet-based interventions for smoking cessation. Cochrane Database Syst Rev. 2013;7:CD007078.

22. Grime PR. Computerized cognitive behavioural therapy at work: a randomized controlled trial in employees with recent stress-related absenteeism. Occup Med (Lond). 2004;54:3539. http://dx.doi.org/10.1093/occmed/kqh077

23. Abbott J, Klein B, Hamilton C, Rosenthal AJ. The impact of online resilience training for sales managers on wellbeing and performance. E-JAP. 2009;5:89-95.

24. Wolever RQ, Bobinet KJ, McCabe K, Mackenzie ER, Fekete E, Kusnick CA et al. Effective and viable mind-body stress reduction in the workplace: a randomized controlled trial. J Occup Health Psychol. 2012;17:246-58. http://dx.doi. org/10.1037/a0027278

25. Martin A, Sanderson K, Cocker F, Hons B. Meta-analysis of the effects of health promotion intervention in the work place on depression and anxiety symptoms. Scand J Work Environ Health. 2009;35:7-18. http://dx.doi.org/10.5271/sjweh.1295

26. Richardson KM, Rothstein HR. Effects of occupational stress management intervention programs: a meta-analysis. J Occup Health Psychol. 2008;13:69-93. http://dx.doi. org/10.1037/1076-8998.13.1.69

27. van der Klink, J J, Blonk RW, Schene AH, van Dijk, F J. The benefits of interventions for work-related stress. Am J Public Health. 2001;91:270-6. http://dx.doi.org/10.2105/ AJPH.91.2.270

28. Cuijpers P, van Straten A, Warmerdam L. Problem solving therapies for depression: a meta-analysis. Eur Psychiatry. 2007;22:9-15. http://dx.doi.org/10.1016/j.eurpsy.2006.11.001

29. D'Zurilla, TJ, Nezu, AM. Problem solving therapies. In: Dobson KS, editor. Handbook of cognitive-behavioral therapies. New York: Guilford Press; 2001. p. 211-245.

30. Kyriacou C. Teacher stress: from prevalence to resilience. In: Langan-Fox J, Cooper CL, editors. Handbook of Stress in the Occupations. Cheltenham: Edward Elgar Publishing; 2011. p. 161-73. http://dx.doi.org/10.4337/9780857931153.00027

31. Lehr D, Hillert A, Keller S. What can balance the effort? Associations between effort-reward imbalance, overcommitment, and affective disorders in German teachers. Int J Occup Environ Health. 2009;15:374-84. http://dx.doi. org/10.1179/oeh.2009.15.4.374

32. Hautzinger M, Bailer M, Hofmeister D, Keller F. [Center for Epidemiological Studies Depression Scale (CES-D; Radloff, L.S., 1977) - German adaptation.] Psychiat Prax. 2012;39:302-4.

33. Radloff LS. The CES-D Scale: A Self-Report Depression Scale for Research in the General Population. Appl Psychol Meas. 1977;1:385-401. http://dx.doi. org/10.1177/014662167700100306

34. Beck AT, Steer RA, Brown, GK. Manual for the Beck Depression Inventory-II. San Antonio, TX: Psychological Corporation; 1996.

35. van Straten A, Cuijpers P, Smits N. Effectiveness of a WebBased Self-Help Intervention for Symptoms of Depression, Anxiety, and Stress: Randomized Controlled Trial. J Med Internet Res. 2008;10:e7. http://dx.doi.org/10.2196/jmir.954

36. Warmerdam L, van Straten A, Twisk J, Riper H, Cuijpers P. Internet-Based Treatment for Adults with Depressive Symptoms: Randomized Controlled Trial. J Med Internet Res. 2008;10:e44. http://dx.doi.org/10.2196/jmir.1094

37. Mohr DC, Cuijpers P, Lehman K. Supportive Accountability: A Model for Providing Human Support to Enhance Adherence to eHealth Interventions. J Med Internet Res. 2011;13:e30. http://dx.doi.org/10.2196/jmir.1602

38. Cuijpers P, Boluijt P, van Straten A. Screening of depression in adolescents through the Internet: Sensitivity and specificity of two screening questionnaires. Eur Child Adolesc Psychiatry. 2008;17:32-8. http://dx.doi.org/10.1007/s00787-007-0631-2 
39. Schwarzer R, Jerusalem M. Generalized Self-Efficacy Scale. In: Wright $\mathrm{S}$, Johnston M, Weinman J, editors. Measures in Health Psychology: A User's Portfolio. Windsor: NFERNelsen; 1995. p. 35-7.

40. Schmitz GS, Schwarzer R. Selbstwirksamkeitserwartung von Lehrern: Längsschnittbefunde mit einem neuen Instrument [Perceived self-efficacy among teachers: Longitudinal findings with a new instrument.]. German Journal of Educational Psychology. 2000;14:12-25.

41. Hinz A, Schumacher J, Albani C, Schmid G, Brähler E. Bevölkerungsrepräsentative Normierung der Skala zur Allgemeinen Selbstwirksamkeitserwartung Bevölkerungsrepräsentative Normierung der Skala zur Allgemeinen Selbstwirksamkeitserwartung [Standardization a scale assessing perceived general self-efficacy in a representative German sample.] Diagnostica. 2006;52:26-32. http://dx.doi.org/10.1026/0012-1924.52.1.26

42. Büssing A, Perrar K. Die Messung von Burnout. Untersuchung einer Deutschen Fassung des Maslach Burnout Inventory [Measurement of burnout. Study of a German version of the Maslach Burnout Inventory (MBI-D).]. Diagnostica. 1992;38:328-53.

43. Iwanicki EF, Schwab RL. A cross-validational study of the Maslach Burnout Inventory. Educ Psychol Meas. 1981;41:1167-74.

44. Fliege H, Rose M, Arck P, Walter OB, Kocalevent R, Weber C et al. The Perceived Stress Questionnaire (PSQ) reconsidered: validation and reference values from different clinical and healthy adult samples. Psychosom Med. 2005;67:78-88. http://dx.doi.org/10.1097/01.psy.0000151491.80178.78

45. Levenstein S, Prantera C, Varvo V, Scribano ML, Berto E, Luzi $\mathrm{C}$, et al. Development of the perceived stress questionnaire: A new tool for psychosomatic research. J. Psychosom. Res. 1993;37:19-32. http://dx.doi.org/10.1016/00223999(93)90120-5

46. Meyer TJ, Miller ML, Metzger RL, Borkovec TD. Development and validation of the Penn State Worry Questionnaire. Behav Res Ther. 1990;28:487-95. http:// dx.doi.org/10.1016/0005-7967(90)90135-6

47. Stöber J, Bittencourt J. Weekly assessment of worry: an adaptation of the Penn State Worry Questionnaire for monitoring changes during treatment. Behav Res Ther. 1998;36:645-56. http://dx.doi.org/10.1016/S0005-7967(98)00031-X

46. D‘Zurilla TJ, Nezu AM, Maydeu-Olivares A. Social Problemsolving Inventory - revised (SPSI-R): Toronto (Ontario): Multi-Health Systems; 2002.

47. GrafA. Psychometrische Überprüfung einer deutschsprachigen Übersetzung des SPSI-R [A Psychometric Test of a German Version of the SPSI-R]. Journal of Individual Differences. 2003;24:277-91.

48. Gandek B, Ware JE, Aaronson NK, Apolone G, Bjorner JB, Brazier JE et al. Cross-Validation of item selection and scoring for the SF-12 Health Survey in nine countries: results from the IQOLA project. J Occup Environ Med. 1998;51:1171-8.

49. Hedeker D, Gibbons RD. Application of random-effects pattern-mixture models for missing data in longitudinal studies. Psychol Methods. 1997;2:64-78. http://dx.doi. org/10.1037/1082-989X.2.1.64

50. Cohen J. Statistical power analysis for the behavioral sciences. 2nd ed. Hillsdale, NJ: L. Erlbaum Associates; 1988.

51. Rosnow RL, Rosenthal R. Assessing the Effect Size of Outcome Research. In: Nezu AM, Nezu CM, editors. Evidence-based outcome research: a practical guide to conducting randomized controlled trials for psychosocial interventions. Oxford, New York: Oxford University Press; 2008. p. 379-401.

52. Jacobson NS, Truax P. Clinical significance: a statistical approach to defining meaningful change in psychotherapy research. J. Consult. Clin. Psychol. 1991;59:12-19. http:// dx.doi.org/10.1037/0022-006X.59.1.12

53. Horwath E. Depressive symptoms as relative and attributable risk factors for first-onset major depression. Arch Gen Psychiatry. 1992;49:817. http://dx.doi.org/10.1001/ archpsyc.1992.01820100061011

54. Lewinsohn PM, Rohde P, Seeley JR. Major depressive disorder in older adolescents: prevalence, risk factors, and clinical implications. Clin Psychol Rev. 1998;18:765-94. http:// dx.doi.org/10.1016/S0272-7358(98)00010-5

55. Schafer JL, Graham JW. Missing data: Our view of the state of the art. Psychol Methods. 2002;7:147-77. http://dx.doi. org/10.1037/1082-989X.7.2.147

56. Beauregard N, Marchand A, Blanc M. What do we know about the non-work determinants of workers' mental health? A systematic review of longitudinal studies. BMC Public Health. 2011;11:439. http://dx.doi.org/10.1186/1471-2458-11-439

57. Spek V, Nyklicek I, Cuijpers P, Pop V. Predictors of outcome of group and internet-based cognitive behavior therapy. J Affect Disord. 2008;105:137-45. http://dx.doi.org/10.1016/j. jad.2007.05.001

58. Warmerdam EH. Online treatment of adults with depression: clinical effects, economic evaluation, working mechanisms and predictors. Amsterdam: Vrije Universiteit; 2010.

59. Ebert DD, Gollwitzer M, Riper H, Cuijpers P, Baumeister H, Berking M. For whom does it work? moderators of outcome on the effect of a transdiagnostic internet-based maintenance treatment after inpatient psychotherapy: randomized controlled trial. J Med Internet Res. 2013;15:e191. http:// dx.doi.org/10.2196/jmir.2511

60. Button KS, Wiles NJ, Lewis G, Peters TJ, Kessler D. Factors associated with differential response to online cognitive behavioural therapy. Soc Psychiatry Psychiatr Epidemiol. 2012;47:827-33. http://dx.doi.org/10.1007/s00127-0110389-1

61. de Graaf LE, Hollon SD, Huibers MJH. Predicting outcome in computerized cognitive behavioral therapy for depression in primary care: a randomized trial. J Consult Clin Psych. 2010;78:184-9. http://dx.doi.org/10.1037/a0018324

62. Federal Statistical Office. Bildung - Lehrkräfte an allgemein bildenden Schulen nach Beschäftigungsumfang und Geschlecht [Education - school teachers in terms of volume of employment and gender]: Federal Statistical Office; 2012 [cited 2012 Nov 9]. Available from: www.statistik-portal.de/ 
Statistik-Portal/de_jb04_jahrtab24sch.asp.

63. Kelders SM, Kok RN, Ossebaard HC, van Gemert-Pijnen JE. Persuasive system design does matter: a systematic review of adherence to web-based interventions. J Med Internet Res. 2012;14:e152. http://dx.doi.org/10.2196/jmir.2104

64. Berking M, Wupperman P. Emotion regulation and mental health: recent findings, current challenges, and future directions. Curr Opin Psychiatry. 2012;25:128-34. http:// dx.doi.org/10.1097/YCO.0b013e3283503669

65. Berking M, Ebert D, Cuijpers P, Hofmann SG. Emotion regulation skills training enhances the efficacy of inpatient cognitive behavioral therapy for major depressive disorder: a randomized controlled trial. Psychother Psychosom. 2013;82:234-45. http://dx.doi.org/10.1159/000348448

66. Heber E, Ebert D, Lehr D, Nobis S, Berking M, Riper H. Efficacy and cost-effectiveness of a web-based and mobile stress-management intervention for employees: design of a randomized controlled trial. BMC Public Health. 2013;13:655. http://dx.doi.org/10.1186/1471-2458-13-655
67. Ebert DD, Lehr D, Smit F, Zarski A-C, Riper H, Heber E et al. Efficacy and cost-effectiveness of minimal guided and unguided internet-based mobile supported stress-management in employees with occupational stress: a three-armed randomised controlled trial. BMC Public Health. 2014:14:807. http://dx.doi.org/10.1186/1471-2458-14-807

68. Bower P, Kontopantelis E, Sutton A, Kendrick T, Richards DA, Gilbody $S$ et al. Influence of initial severity of depression on effectiveness of low intensity interventions: meta-analysis of individual patient data. BMJ. 2013;346:f540. http://dx.doi. org/10.1136/bmj.f540

69. Rozental A, Andersson G, Boettcher J, Ebert DD, Cuijpers $\mathrm{P}$, Knaevelsrud $\mathrm{C}$ et al. Consensus statement on defining and measuring negative effects of Internet interventions. Internet Interventions. 2014;1(1), 12-19. http://dx.doi.org/10.1016/j. invent.2014.02.001

70. Ebert DD, Lehr D, Baumeister H., Boß L, Riper H, Cuijpers P, et al. GET.ON Mood Enhancer: efficacy of Internet-based guided self-help compared to psychoeducation for depression: an investigator-blinded randomised controlled trial. Trials. 2014;15(1), 39. http://dx.doi.org/10.1186/1745-6215-15-39

Received for publication 25 December 2013 